
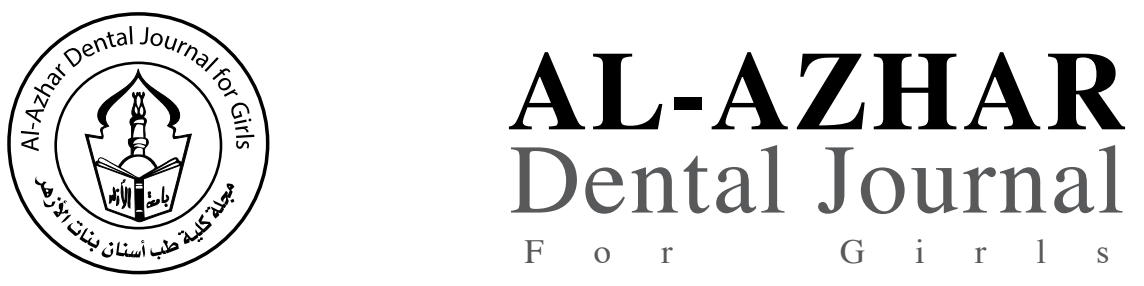

The Official Publication of The Faculty of Dental Medicine For Girls,

Al-Azhar University Cairo, Egypt.

ADJ-for Grils, Vol. 4, No. 1, January (2017) — PP. 63:70

\title{
Effect of Different Preparation Designs on the Fracture Load of Two Machinable Laminate Veneers
}

\section{Sara Tamimi ${ }^{(1)}$ Eman Ahmed Essam ${ }^{(2)}$ and Jylan Fouad El Guindy ${ }^{(3)}$}

Codex : 09/1701

dentaljournal.forgirls@yahoo.com

\section{KEYWORDS}

Laminate veneer,

Translucent zirconia

Lava ultimate resin nano

ceramics.

\begin{abstract}
Aim: The present study was performed to evaluate the effect of different preparation designs [window, butt- Joint, and incisal overlap], on the fracture load of two CAD / CAM laminate veneer materials [Zirconia CAD / CAM and resin nanoceramic ; lava ultimate].Materials and Methods: Thirty maxillary anterior teeth were restored with ceramic laminate veneers and divided into 6 groups as follows: group I-A (laminates made of Ultra translucent zirconia CAD with window preparation design), group I-B (laminates made of Ultra translucent zirconia CAD with butt-joint preparation design), group I-C (laminates made of Ultra translucent zirconia CAD with incisal overlap preparation design), group II-A (laminates made of Lava ultimate (Resin nano ceramic) CAD with window preparation design), group II-B (laminates made of Lava ultimate CAD with butt-joint preparation design), group II-C (laminates made of Lava ultimate CAD with incisal overlap preparation design). Fracture load test was performed using the Universal Testing Machine (UTM). Data were statistically analyzed using One-way analysis of variance ANOVA followed by Tukey's multiple comparison test. The results of the fracture load test were recorded in Newton: (I-A: $288.08 \mathrm{~N}$ ); (I-B: 322.47N); (I-C: 365.38N); (II-A: 301.15N); (II-B: 324.94N); (II-C: 394.95N). The results of the current study showed that there was no statistically significant difference between the means of fracture load for window, butt-joint and incisal overlap preparation designs among zirconia material. While, there was statistically highly significant difference between the means of fracture load for window, butt-joint and incisal overlap preparation designs among Lava ultimate material. The mean of fracture load for incisal over-lap preparation design was the highest, followed by butt-joint and window designs. Nonetheless, there were no statistically significant differences between translucent zirconia and lava ultimate regarding the fracture load among window, butt-joint and incisal overlap preparation designs.
\end{abstract}

Paper extracted from Master thesis entitled" Effect of Different Preparation Designs on the Fracture Load of Two Machinable Laminate Veneers"

1. Demonstrator, Crowns and Bridges Department. Faculty of Dental Medicine for Girls, AL-Azhar University.

2. Associate Professor of crowns \&Bridges. Faculty of Dental Medicine for Girls, AL-Azhar University.

3. Professor of Fixed Prosthodontics. Faculty of Oral and Dental Medicine, Cairo University. 


\section{INTRODUCTION}

Esthetic or cosmetic dentistry has become one of the main areas of dental practice emphasis and growth for several years. Recently the main reason for applying restorative dental materials is not only to restore dental tissues lost because of caries or trauma, but also to correct the form and color of teeth for social acceptance. During the past two decades, porcelain laminate veneers have proved to be a reliable and successful technique for discolored, malformed, worn, or fractured teeth, especially in visible areas. Dental ceramics can both improve the esthetic appearance and reestablish the strength and function of teeth. In worn anterior teeth, functional surfaces, for example, anterior and lateral guidance, can be restored effectively. The characteristics of dental ceramics, such as color stability and mechanical and optical properties, make this material a good choice for indirect restorations, especially when optimum function and esthetics are required ${ }^{(1-2)}$.

Tooth preparations for porcelain laminate veneers are crucial for optimal function and esthetics. Clinical cohesive ceramic fractures have occurred mainly at the incisal edge of the veneer because of greater stress. It was believed that a palatal chamfer was necessary to strengthen ceramic veneers. Unfortunately, most of the data regarding the clinical behavior of different tooth preparation designs originated from anecdotal reports. It remains controversial, whether different tooth preparation designs can affect fracture strength of ceramic veneers or whether one configuration of tooth preparation is superior to another. Hence, attempt was made to study and compare the fracture resistance of ceramic veneers with three different incisal preparations ${ }^{(3)}$.

\section{MATERIALS AND METHODS}

A total of thirty caries-free human maxillary central incisors were selected for this study. The teeth were examined for being approximately equal in the mesio-distal and inciso- cervical dimentions at the coronal portion. The samples were divided into two main groups $(1 \& 2)$ according to the ceramic material used for construction of laminate veneers: 1- Fifteen teeth for lava ultimate CAD restorative material. 2- Fifteen teeth for translucent zirconia CAD ceramic material. Each group was sub-divided into three equal subgroups $(\mathrm{A}, \mathrm{B}, \mathrm{C})$, according to different preparation designs. (window, butt-joint, and incisal overlap preparation designs), as shown in table (1).

Table (1): Groups and subgroups of this study.

\begin{tabular}{|c|c|c|}
\hline $\begin{array}{c}\text { Preparation } \\
\text { Designs }\end{array}$ & $\begin{array}{c}\text { Lava ultimate } \\
\text { CAD (group 1) } \\
\text { (15 samples) }\end{array}$ & $\begin{array}{c}\text { Translucent zirco- } \\
\text { nia CAD (group } 2) \\
(15 \text { samples })\end{array}$ \\
\hline Window & A (5 samples) & A (5 samples $)$ \\
\hline Butt-joint & B (5 samples) & B (5 samples $)$ \\
\hline Incisal overlap & C (5 samples $)$ & C (5 samples $)$ \\
\hline
\end{tabular}

The teeth were mounted individually in special plastic cylinder molds with epoxy resin, with the long axis parallel to the center of the mold. Each tooth was suspended in the middle of the mold using a Ney Surveyor to ensure vertical positioning of inside the mold. All specimens were embedded up to $2 \mathrm{~mm}$ below the cemento-enamel junction to simulate the natural biologic width. To standardize the amount of reduction; a silicone index was made. The putty consistency of condensation silicone impression material was used for taking the index of all the teeth before and checking after reduction. Standardized preparations were done in the labial surface of all teeth using a 3 wheel depth cutter diamond stone. By using this Standardized diameter instrument, equal preparations of approximately $0.5 \mathrm{~mm}$ depth have been performed.

Labial reduction was $0.5 \mathrm{~mm}$ to ensure that the whole preparation remained in enamel. The reduction was carried out at two different planes 
to follow the contour of the labial surface. This was done using a tapered diamond stone with a round end. The cervical margin was placed $1 \mathrm{~mm}$ coronal to the cement-enamel junction. A chamfer finish line was insured all around the preparation margin; it was checked for uniformity and continuity. The thickness of the finish line was checked using a digital caliper. Proximal reduction was done using the tapered diamond stone with round end. The end of the proximal reduction was placed just beyond the mesio-labial and disto-labial line angles. The entire reduction was completed within the enamel.

For the window preparation: teeth were prepared labially close to but not up to the incisal edge. For the butt-joint preparation: teeth were prepared with a $2 \mathrm{~mm}$ incisal reduction without palatal chamfer. For the Incisal overlap preparation: teeth were prepared with a $2 \mathrm{~mm}$ incisal reduction and $1 \mathrm{~mm}$ height palatal chamfer. Any sharp angles that might serve as a focal point for stress concentration were removed,particularly at the junction of the incisal line and point angles to both the labial and lingual surfaces using a finishing diamond stone.

To obtain a three dimensional image for each specimen on the computer screen, the following steps were performed: The prepared teeth were sprayed using light reflecting powder for taking the optical impression.An automatic margin finder is used for preparation margin detection. Ceramic laminate veneer thickness was checked by the software in order to standardize the thickness of all the samples and then next icon was clicked so that the finished restoration was displayed in the milling situation. After completion of the milling process, the veneers were separated manually from either zirconia disk or lava ultimate blocks with a diamond cutting instrument. According to manufacturer's instructions, The Lava Ultimate Restorative veneers didn't require any further firing or glazing. As ultra translucent zirconia disks were initially manufactured in partially sintered state, so the roland CAD/CAM milling machine is programmed to produce an enlarged restoration with a percentage equal to that of the shrinkage percent during sintering process. The laminates were then placed inside the ceramic sintering tray and then sintered in the HTC furnace (High temperature furnace) with program control unit. After sintering, zirconia laminate veneers were stained and glazed using Cerabien ZR FL glaze, VC glaze and Noritake ES liquid, then the zirconia laminate veneers were inserted into the Programat P300 furance for glaze firing. Then all laminate veneers were seated on their corresponding teeth and checked for complete seating. The fitting surfaces of ceramic laminate veneers were sandblasted with aluminum oxide 50 um particle size. All laminate veneers were primed for resin on their intaglio surface using a single bond universal adhesive. The adhesive was applied to the laminate veneer and rubbed for 20 seconds. The adhesive was gently air dried for approximately 5 seconds to evaporate the solvent. Cementing the restoration according to manufacturer's instructions was then performed. Surface treatment of enamel was also done using $32 \%$ scotchbond universal etchant for 15 -second. Then Single bond universal adhesive was applied on the etched enamel surfaces and rubbed for 20 seconds, gently air dried for approximately 5 seconds to evaporate the solvent, then light cured for 10 seconds.

A dual cure composite resin luting agent (Rely $x$ ultimate) was used to lute the veneers. Light curing was performed 20 seconds for each surface. All the samples were stored in saline solution at room temperature $37^{\circ} \mathrm{C}$ until fracture load testing. All samples were individually mounted on a computer controlled materials testing machine (Model 3345; Instron Industrial Products, Norwood, MA, USA). Fracture test was done by compressive mode of load applied at $135^{\circ}$ angle using a metallic rod with flat tip ( $5 \mathrm{~mm}$ diameter). The load at failure was manifested by an audible crack and confirmed by a sharp drop at load-deflection curve recorded using computer software (Instron ${ }^{\circledR}$ Bluehill Lite Software). The load required to fracture was 
recorded in Newton. Mode of failure of all samples was assessed using magnifying loupe. One sample, representing each mode of failure was randomly selected and scanned under electron microscope (SEM).

\section{RESULTS}

The fracture load values and standard deviations for all samples are indicated in table (2). In group I (ultra translucent zirconia), Incisal overlap design showed the highest values (365.38), followed by Buttjoint design (322.47) and the lowest values were observed in the window design (288.08). In group II (lava ultimate), Incisal overlap design showed the highest values (394.95), followed by Buttjoint design (324.94) and the lowest values were observed in the window design (301.15). One- way analysis of variance ANOVA followed by Tukey's multiple comparison test were used to evaluate the significance between subgroups, separate analysis was performed with t- test between subgroup. A two factorial analysis of variance ANOVA was used to examine effects of material $\mathrm{v}$ preparation designs and the interactions between these factors. There was no statistically significant difference between the means of fracture load for window, Butt-joint and incisal overlap preparation designs among zirconia material. But there was statistically highly significant difference between the means of fracture load for window, Butt-joint and incisal overlap preparation designs among Lava ultimate material.

Table (2) One-way ANOVA multiple comparisons between different designs regard fracture load among zirconia and lava ultimate.

\begin{tabular}{|c|c|c|c|c|c|c|c|}
\hline Material & Design & $\mathrm{N}$ & Mean & SD & F & P Value & Sig. \\
\hline \multirow{3}{*}{ Zirconia } & Window & 5 & 288.08 & 44.72 & \multirow{3}{*}{3.20} & \multirow{3}{*}{0.077} & \multirow{3}{*}{ NS } \\
\hline & Butt-joint & 5 & 322.47 & 52.35 & & & \\
\hline & Incisal overlap & 5 & 365.38 & 47.82 & & & \\
\hline \multirow{3}{*}{ Lava } & Window & 5 & 301.15 & 13.14 & \multirow{3}{*}{32.84} & \multirow{3}{*}{0.000} & \multirow{3}{*}{ HS } \\
\hline & Butt-joint & 5 & 324.94 & 25.11 & & & \\
\hline & Incisal overlap & 5 & 394.95 & 16.82 & & & \\
\hline
\end{tabular}

\section{DISCUSSION}

In the present study, human central incisor teeth were selected for specimen fabrication because their bonding characteristics, thermal conductivity, modulus of elasticity and strength represent the clinical condition better than would plastic or animal teeth ${ }^{(4-7)}$.
A uniform reduction of $0.5 \mathrm{~mm}$ of the labial surface was performed to ensure that the whole preparation was confined into enamel, which is a recommended situation to allow better bonding, higher strength, less leakage, and excellent color matching without over contouring ${ }^{(5,8)}$.

Three preparation designs for laminate veneers were selected in this study, the designs were: 
(A) Window preparation design; teeth were prepared labially, but the incisal edge height was not reduced. (B) Butt-joint preparation design; teeth were prepared with a $2 \mathrm{~mm}$ incisal reduction without palatal chamfer. (C) Incisal overlap preparation design; teeth were prepared with a $2 \mathrm{~mm}$ incisal reduction and $1 \mathrm{~mm}$ height palatal chamfer ${ }^{(5,8-9)}$.

In the present study, laminate veneers were fabricated from two ceramic materials; Ultra Translucent zirconia and Lava ultimate CAD/ CAM restorative materials. Resin nanoceramic (Lava $^{\mathrm{TM}}$ Ultimate) is a recently introduced unique CAD/CAM block based on the integration of nanotechnology and ceramics. This nano resin ceramic material is purported to offer the ease of handling of a composite material with the surface gloss and finish retention similar to porcelain.

The CAD /CAM technology was used in this study, the in- lab milling system. The teeth were fixed to the tray of the scanner using specific clay. Then the prepared teeth were sprayed using light reflecting powder to be scanned using the Jscan scanner for taking the optical impression. The scanning process of the tooth was completed after 14 minutes and a digital impression was captured for the tooth. Then the captured picture was saved in the occlusion catalogue of the software. The software designed the ceramic laminate veneers which were milled by the Roland machine. 'Roland' CAD/CAM system used in the present study is 5-axis milling machine, the milled blocks/discs are rotated $360^{\circ}$ in both clockwise and counter clockwise directions allowing for materials to be tilted forward and backward $20^{\circ}$ supporting complex cuts. Moreover; it has five station automatic tool changer with tool length sensor. The Roland system was used because it is an open system that can be used with both ultra translucent zirconia disks and Lava ultimate blocks ${ }^{(\mathbf{1 0})}$.

Treating the bonding surface of zirconia with 50 um $\mathrm{AL}_{2} \mathrm{O} 3$ resulted in high values of shear bond strength that may be attributed to the fact that treating zirconia bonding surface with sandblast increases surface roughness and undercuts. Furthermore it produced significant enhancement in bonding strength. The use of sandblast with Primer gave the highest mean of shear bond strength.

When Lava Ultimate CAD/CAM is used, the surface treatment of sandblasting is suggested also in order to get a better bonding. Therefore sandblasting the intaglio surface of both zirconia and lava ultimate laminate veneers was the treatment option in this study ${ }^{(6,11-13)}$. All laminate veneers were primed for resin on their intaglio surface using a single bond universal adhesive (Adhesive and primer in one bottle). The adhesive was applied to the laminate veneer and rubbed for 20 seconds. The adhesive was gently air dried for approximately 5 seconds to evaporate the solvent. Cementing the restoration according to manufacturer's instructions was then performed $^{(6,11)}$.

Total etch technique was used for conditioning the prepared teeth using Scotchbond universal etchant then single bond universal adhesive to enhance the bonding procedure. In this study, Rely x Ultimate, dual cure adhesive resin cement was used for cementation of laminate veneers to their corresponding prepared teeth. This cement offers ultimate bond strength, tooth-like fluorescence, high wear resistance, Consistent bond strength to both moist- and dry-etched dentin. It also combined total- etch and self-etch bonding capability and integrated primers for glass ceramics, oxide ceramics and metal ${ }^{(14)}$.

Regarding the three preparation designs, the present study showed that there was statistically highly significant difference between the means of fracture load for window, Butt-joint and incisal overlap designs. The mean of fracture load for incisal overlap preparation design was the highest mean, followed by butt-joint and window designs regardless of the ceramic materials (translucent zirconia CAD and Lava ultimate CAD CAM restorative material) used. Our results 
come in agreement with $\mathrm{Li}$ et al ${ }^{(9)}$; they stated that the preparation of a palatal chamfer increased the volume of the veneer. As a result, a larger restoration might distribute stress more uniformly, So a palatal chamfer design might be a better choice for porcelain veneers because it has a lower maximum principle stress, a more uniform stress distribution in the cement layer, and a high clinical success rate.

Our findings were also in agreement with Jankar et al ${ }^{(8)}$; they found that among the three groups, group III $(1 \mathrm{~mm}$ incisal reduction with $1 \mathrm{~mm}$ height of palatal chamfer) had the highest fracture resistance and is stronger than group I (No incisal reduction with facio incisal bevel) and II (1mm incisal reduction with butt joint). However, statistically no significant difference was found between Group II and III. The increase in the fracture resistance was due to an increase in available bonding surface area. The $1 \mathrm{~mm}$ incisal reduction and rounded incisal edge accentuate the bucco-lingual width and palatal chamfer develops a bound at right angle to the direction of potential displacement of the tooth. This design prevents the torque of the incisal porcelain to the underlying tooth surface. The resultant fracture is seen usually at the junction of the labial and incisal plane. The palatal chamfer margin result in preservation of some peripheral enamel layer, which eliminates the micro leakage at the palatal margin-restoration interface and also effectively counteracting shear stress. This design provides a definite seat for cementation. Group II (1mm incisal reduction with butt joint) recorded fracture resistance greater than Group I (No incisal reduction with facioincisal bevel) and the difference was statistically significant. Butt-joint incisal configuration still permits the preservation of peripheral enamel layer around all margins.

Conversely, some authors ${ }^{(15)}$ reported a decrease in mean dental fracture strength of $76.53 \mathrm{~N}$ in teeth with feathered incisal edge preparations, and 102.82 $\mathrm{N}$ for those with palatal chamfers when compared with non prepared teeth, whereas the preparation with a butt joint showed no significant difference compared to non prepared teeth. Ceramic fractures occurred more frequently in the chamfer type than feathered incisal edge design. So, the butt joint was the type of preparation that least affected the strength of the tooth and the chamfer preparation type was more susceptible to ceramic fractures. Other researchers ${ }^{(5,16)}$ preferred the buttjoint design for laminate veneers, because the window design was claimed to lead to inadequate veneer seating, increased marginal discrepancies, staining and porcelain chipping. Also extending the preparation in to the palatal surface in the form of the incisal overlap preparation design created a thin ceramic shell in an area of maximum tensile stresses that showed to be a possible cause of failure. These results may be attributed to the use of different ceramic materials.

Regarding the two ceramic materials used in this study, There were no statistically significant differences between zirconia $(325.31 \mathrm{~N})$ and lava ultimate $(340.35 \mathrm{~N})$ regarding the fracture load among window, Butt-joint and incisal overlap preparation designs.

Our results were within the clinical acceptance range with Abdul Khaliq et al ${ }^{(6)}$ who found that the fracture strength of Lava ultimate laminate veneers was $271.8 \pm 68.796$ N.The fracture strength values obtained for teeth restored with indirect composite and resin nano ceramic veneers confirm the theory that polymer materials have greater capacity to distribute tensions in a more homogeneous way than ceramics as they present greater resiliency resulting in a larger capacity to buffer plastic deformations, preserving the adhesive interface. Another important aspect that explains this point is the synergism of behavior among the indirect resins, resin cement and adhesive system, which have similar compositions and high bond capacity among themselves ${ }^{(\mathbf{1 7})}$.

The mean failure loads of the groups in this in vitro study ranged from $(288.08 \mathrm{~N})$ to $(394.95 \mathrm{~N})$ 
reaching levels higher than the physiological biting force of the anterior teeth which somewhat varies between $155 \mathrm{~N}$ and $200 \mathrm{~N}^{(18-19)}$ concluding that both types of ceramic materials and all three different preparation designs of laminate veneers used could be considered strong enough to withstand anterior forces. Our results proved that Lava ultimate resin nano ceramic is more sensitive to the preparation designs, while translucent zirconia is not significantly affected by the preparation designs. This may be attributed to polymer materials have greater capacity to distribute tensions in a more homogeneous way than ceramics as they present greater resiliency resulting in a larger capacity to suffer plastic deformations, preserving the adhesive interface. Another important aspect that explains this point is the synergism of behavior among the indirect resins, resin cement and adhesive system, which have similar compositions and high bond capacity among themselves. ${ }^{(17)}$ On the other hand, the increased stiffness of the tooth and Zirconia laminate veneers' causing stress concentration in the cervical area ${ }^{(20)}$. The cervical fractures of incisors under static loading conditions were a common observation, as reported in other previous studies (20-21). So zirconia laminate veneers were not affected by the preparation designs because most of the stresses were transmitted to the cervical area and this explained why more than $75 \%$ of the failure modes of zirconia groups in our study were cervical tooth fracture (22). Thus the null hypothesis of this study was accepted regarding the two different $\mathrm{CAD} / \mathrm{CAM}$ materials, and was rejected regarding the preparation designs.

\section{CONCLUSIONS}

Within the limitations of this study, the following conclusions could be drawn:

- The fracture load of Lava ultimate resin nano ceramic material is more sensitive to the preparation design.
- The fracture load for incisal over-lap preparation design is the highest, followed by butt-joint and window designs irrespective of ceramic material used.

- The fracture load of laminate veneers made of both Lava ultimate resin nano ceramic and zirconia CAD/CAM ultra translucent materials is comparable; and exceeds the physiologic anterior biting forces.

\section{RECOMMENDATIONS}

Further investigations should be done to improve the bond strength between zirconia and adhesive resin cement.

\section{REFERENCES}

1. Belser U, Magne P, Magne M. Ceramic Laminate veneers: continuous evolution of indications. J Esthet Dent 1997; 9: 197-207.

2. Da Cunha LF, Pedroche LO, Gonzaga CC, Furuse AY. Esthetic, occlusal, and periodontal rehabilitation of anterior teeth with minimum thickness porcelain laminate veneers. J Prosthet Dent 2014; 112: 1315-18.

3. Garber DA. Rational tooth preparation for porcelain laminate veneer compared. Compendium. 1991; 12:316-20.

4. Aboushelib M N, Elmahy W A, Ghazy M H. Internal adaptation, marginal accuracy and microleakage of a pressable versus a machinable ceramic laminate veneers. Journal of dentistry.2012; 40:670-7.

5. Khatib D, Katamish H, Ibrahim AS. Fracture load of two cad/cam ceramic veneers with different preparation designs. C.D.J. 2009; 25: 425-32

6. Abdul Khaliq A G, Al-Rawi I I. Fracture strength of laminate veneers using different restorative materials and techniques. (Acomparative in vitro study). J Bagh Coll Dentistry. 2014; 26:1-8.

7. Duzyol M, Duzyol E, Seven N. Fracture Resistance of Laminate Veneers Made with Different Cutting and Preparation Techniques. International Journal of Dental Sciences and Research, .2016; 4:42-8.

8. Jankar AS, Kale Y, Kangane S, Ambekar A, Sinha M. et al. Comparative evaluation of fracture resistance of Ceramic Veneer with three different incisal design preparations - An In-vitro Study. J Int Oral Health.2014; 6: 48-54. 
9. Li Z, Yang Z, Zuo L, Meng Y. A three-dimensional finite element study on anterior laminate veneers with different incisal preparations. J Prosthet Dent 2014; 112:325-33.

10. ETI Empire direct. Open CAD CAM systems. Roland milling machines for digital dentistry.2016; 1451-7778.

11. Dawood RM, Ibraheem AF. Evaluation of shear bond strength of zirconia to tooth structure after different zirconia surface treatment techniques. J Bagh coll dentistry.2015; 27: 77-85.

12. Chen C, Trindade FZ, Jager ND, Kleverlaan CJ, Fielzer AJ. The fracture resistance of a CAD/CAM Resin NanoCeramic (RNC) and a CAD ceramic at different thicknesses. Dental materials.2014; 30:954-62.

13. Cavalcanti AN, Foxton RM, Watson TF, Oliveira MT, Giannini M, Marchi GM. Bond strength of resin cements to a zirconia ceramic with different surface treatments. Operative Dentistry 2009; 34: 280-7.

14. 3M Dental products: Rely X ARC (Adhesive resin cement system) Brochure.

15. Da Costa DC, Coutinho M, De Sousa AS, Ennes JP. A meta-analysis of the most indicated preparation design for porcelain laminate veneers. J of Adhesive Dent. 2013, 15:215-20.
16. Smales R, Etemadi S.: Long term survival of porcelain laminate veneers sing two preparation designs: A retrospective study. Int. J. Prosthodont.2004; 17: 323-26.

17. Soares CJ, Martins LR, Pfeifer JM, Giannini M. Fracture resistance of teeth restored with indirect composite and ceramic inlay systems. Quintessence Int 2004; 35: 281-6.

18. Hahn P, Gustav M, Hellwig E. An in vitro assessment of the strength of porcelain veneers dependent on tooth preparation . J Oral Rehabil. 2000; 27: 1042-49.

19. Gresnigt M, Ozcan M. Fracture strength of direct versus indirect laminates with and without fiber application at the cementation interface. Dent Mater.2007; 23: 927-33.

20. Castelnuovo J, Tjan AH, Philips K, Nicholls JI, Kois JC. Fracture load and mode of failure of ceramic veneers with different preparations. J Prosthet Dent. 2000; 83: 171-80.

21. Stappert CFJ, Ozden U, Gerds T, Strub JR. Longevity and failure load of ceramic veneers with different preparation designs after exposure to masticatory simulation. J Prosthet Dent 2005; 94: 132-39.

22. Turkaslan S, Tezvergil M, Bagis B, Shinya A, Vallittu P K. Effect of Intermediate Fiber Layer on the Fracture Load and Failure Mode of Maxillary Incisors Restored with Laminate Veneers. J Dent Mater. 2008; 27-61-8. 